species into consideration. Data integration and implementation of human tissues and cells in cell culture has great importance in current scientific work and increases predictive power of experiments analogous to animal experiments. In vitro methods often have the disadvantage that they need to be validated against animal experiments, the so-called "gold standard", which may not be the best choice for an extrapolation to human data. In vitro models have their strengths in elucidating molecular mechanisms and can offer added value for defined questions.

\section{Bettina Seeger}

Institute for Food Toxicology and Virtual Center for Replacement/

Complementary Methods to Animal Testing

University of Veterinary Medicine, Hannover, Germany

\title{
Meeting Report The Biological Evaluation of Medical Devices:
Transition to 2017/745 MDR in Progress
}

doi:10.14573/altex.1907011

On May 23, 2019 VitroScreen organized a seminar on the biological evaluation of medical devices (MD) focusing on biocompatibility aspects (cytotoxicity, irritation, and sensitization potential). MDs are an heterogenous and complex class of products whose legislative framework has recently been revised with the introduction of EU Regulation 2017/745 (MDR). Being a key step within the assessment and risk management process, the biological evaluation has to be properly addressed and documented in the dossier of each device entering the market. Currently, there are no harmonized standards and common specifications available to drive the transition from the old MD Directive 93/42/CEE to the new MDR and a large number of questions have arisen from industry as well as from the Notified Bodies involved in the CE marking process.

Dr Marisa Meloni, CEO of VitroScreen, welcomed the participants and highlighted the importance of gathering together representatives of Notified Bodies (ISS, ITALCERT, Kiwa Cermet, and others), as well as toxicologists and regulatory toxicology experts, members of the ISO/TC 194 Working Group, and regulatory affairs specialists operating in the industry and academia in a think tank to address hazard and risk assessment issues in the medical device sector. This allowed to discuss the difficulties being encountered, to share experiences and expertise, and to formulate a vision for the future of medical device evaluation in order to move forward towards a robust toxicological approach by overcoming the difficulties and uncertainties in the transition period. The seminar offered a unique opportunity to achieve a better understanding of the evolution of the ISO 10993 standard and the applicability of OECD validated in vitro test methods and non-animal testing approaches to the assessment of the skin irritation and skin sensitization potential of medical device extracts and substance-based devices. It was underlined that the ISO standard is evolving not only to respond to regulatory requirements but also to the ethical and scientific concerns which demand the replacement of animal testing and the adoption of more reliable and predictive testing strategies according to Directive 2010/63/ EU.

Dr Christian Pellevoisin (EpiSkin, Convenor of ISO Group 8, Member of Technical Committee 194 (ISO/TC 194/WG8)) delivered a lecture entitled "Updates to future ISO 10993-23 standard: in vitro assessments to support biocompatibility and perspectives for sensitizing potential" underlining the significant progress achieved in the evaluation of skin irritation and skin sensitization potential in the medical device sector at ISO level. Skin irritation testing is one of three biocompatibility tests recommended for all medical devices (ISO 10993-1), the others being skin sensitization and cytotoxicity. According to ISO 10993-10 "Biological evaluation of medical devices - Part 10: Tests for irritation and skin sensitization", some in vitro approaches do exist for this endpoint but they are only validated for neat chemicals and not for medical device extracts. Hence, the Draize test performed in vivo on rabbits is still requested. Knowing both the ethical and scientific limits of animal approaches, the ISO TC194/WG8 initiated a project to adapt the existing in vitro OECD protocol for chemicals (TG 439) to medical device (MD) products in 2012. The OECD TG 439 for skin irritation of chemicals is based on reconstructed human epidermis models $(\mathrm{RhE})$. Validated $\mathrm{RhE}$ models have undergone long and strict evaluation processes by centers of validation of alternative methods (ECVAM, JaCVAM) before their acceptance. The robustness, quality, and stability of the RhE models as well as the transferability and predictability of the methods have been challenged with large sets of chemicals in at least 3 different laboratories. Then, the results were peer-reviewed by an independent scientific committee before being considered for inclusion in TG 439. Considering this and the recent guideline for good in vitro methods from OECD (GIVIMP), it has been decided to rely only on RhEs previously validated in an OECD TG to develop a protocol adapted to medical devices. Two models, SkinEthic RHE from EPISKIN (FR) and Epiderm from MatTek (US) have been used to develop a method to assess 
skin irritation potential of medical device extracts. After optimization and transfer to naïve laboratories, a blinded round robin study was performed in 17 laboratories, which tested 7 reference materials ( 4 irritants, 3 non irritants) on 3 different RhE batches. The statistical analysis of the results revealed an excellent within/ between variability and a predictivity close to $100 \%$. The results were published in a special edition of Toxicology In Vitro in 2018. Based on these results, the WG8 decided to split the former ISO 10993-10, keeping skin sensitization in part 10 and creating ISO 10993-23 for irritation endpoints. The new, stepwise approaches to assess skin irritation rely now first on in vitro skin irritation testing with RhE before envisaging any further in vivo assay, if necessary. The applicability domains of the validated in vitro assays focus mainly on MD extracts but can be extended to non-extractable samples, i.e., as substance-based MD, when suitability is justified. ISO 10993-23 is at the final discussion stage before release. For skin sensitization, there are also ethical and scientific reasons to shift from animal to alternative methods as the accuracy of the commonly used animal tests (GPMT, Buehler test, LLNA) barely reaches $70 \%$ compared to human data. In 2012, based on international scientific consensus, OECD proposed an Adverse Outcome Pathway (AOP) for skin sensitization with four key events. Since then, several in chemico and in vitro assays have been validated to be used in testing strategies. However, these OECD assays concern neat chemicals and use mainly cell lines cultivated in $2 \mathrm{D}$, which are neither easily adapted to medical device extracts nor to complex mixtures in which potential leached sensitizers are diluted in polar and non-polar solvents. This toxicological endpoint will be discussed in the coming meetings of the WG8 to evaluate how the testing strategy used for pure chemicals could be adapted to the specific context of MD. Unlike for skin irritation, much quantitative data for skin sensitization has been generated from human (NOAEL/NESIL) or animal data (EC3). This will facilitate the creation of reference test samples to robustly assess the performance of adapted OECD methods to the context of medical device products.

Dr Roberta Marcoaldi and Dr Roberta Feliciani (Notified Body 0373, Istituto Superiore di Sanità) in their presentation entitled "Esperienze di un Organismo Notificato sugli studi di biocompatibilità" (Experiences of a Notified Body on biocompatibility studies) pointed out that the procedure for the biological evaluation of medical devices is of crucial importance in the context of the CE marking process and is part of a broader assessment and risk management program that manufacturers follow and for which they must provide documented evidence to the Notified Body for CE marking purposes. From the legislative point of view, Directive 93/42/CEE and subsequent changes show specific requirements in terms of biological evaluation, and Directive 2007/47/EC has underlined the need to include the documentation relating to the preclinical evaluation in the technical file prepared by the medical device manufacturer. The MDR also reaffirms what has already been provided for in Directive 93/42/EEC in terms of biological evaluation of medical devices. Biocompatibility tests are chosen based on material categorization and on the nature and duration of contact with the patient's body; they must be conducted on the finished and sterilized medical device (if supplied in a sterile form) in compliance with the harmonized standard ISO 10993, and they must be selected based on the final use of the device. If a medical device is equivalent to devices already on the market and the manufacturer demonstrates, in the case of substance-based medical devices, that the components used in the new medical device are the same, are present in the same quantities, and have the same functionality and purpose within the formulation compared to the equivalent device on the market, the Notified Body can accept a biological evaluation carried out on the new device by the manufacturer based on literature and/or market data referring to the equivalent device without requiring biocompatibility tests. Finally, the manufacturer must give evidence to the Notified Body of the management of changes with respect to the biocompatibility requirement of medical devices already approved by the same Body. If the manufacturer has not carried out biocompatibility tests or new tests, or there are deviations from the procedures, the Notified Body has to examine the motivation presented by the manufacturer.

Prof. Emanuela Corsini (Milan University) delivered a very interesting and stimulating lecture entitled "La valutazione del potenziale sensibilizzante: metodi in vivo ed in vitro a confronto" (Evaluation of sensitizing potential: comparison between in vivo and in vitro methods), highlighting the relevance of using validated methods and methods under validation in the field of skin sensitization and their applicability to the assessment of finished products such as medical devices. According to the new MDR, all medical devices must undergo sensitization testing if direct or indirect contact with the body is expected. Medical devices that cause sensitization reactions do so by means of their extractable chemicals. Currently, in vivo and in vitro methods have been developed to test pure substances. Among the animal models, the Guinea pig methods are generally preferred to the mouse local lymph node assay (LLNA) to test medical devices. The Magnuson-Kligman maximization test is considered more sensitive than the Buehler test. It is used for those devices that will contact areas other than the skin and involves the use of both a water extract and an oil extract to simulate extraction with body fluids and intravenous fluids that will come into contact first with the device and then the patient. On the contrary, the Buehler test is mainly used for topical devices, such as dermal electrodes, lab coats and surgical drapes, since in these cases the method of applying test materials to animals simulates clinical use. Regarding the use of the recently validated non-animal tests, only few studies have been published on their use to assess medical devices, and their applicability to medical device extracts still needs to be demonstrated. One main problem is to define the sensitivity that the in vitro method should have to identify the release of potential allergens, both for hazard identification and risk assessment, as the threshold concept is controversial and could be challenging using in vitro methods. Potential sensitizers are often extremely diluted and mixed with other substances in medical device extracts. For some of the validated in vitro methods addressing key events 2 and 3 of the AOP leading to skin sensitization, like the KeratinoSens, hCLAT, LuSens, MUSST, and IL-8 Luc assays, we can foresee the possibility to test water 


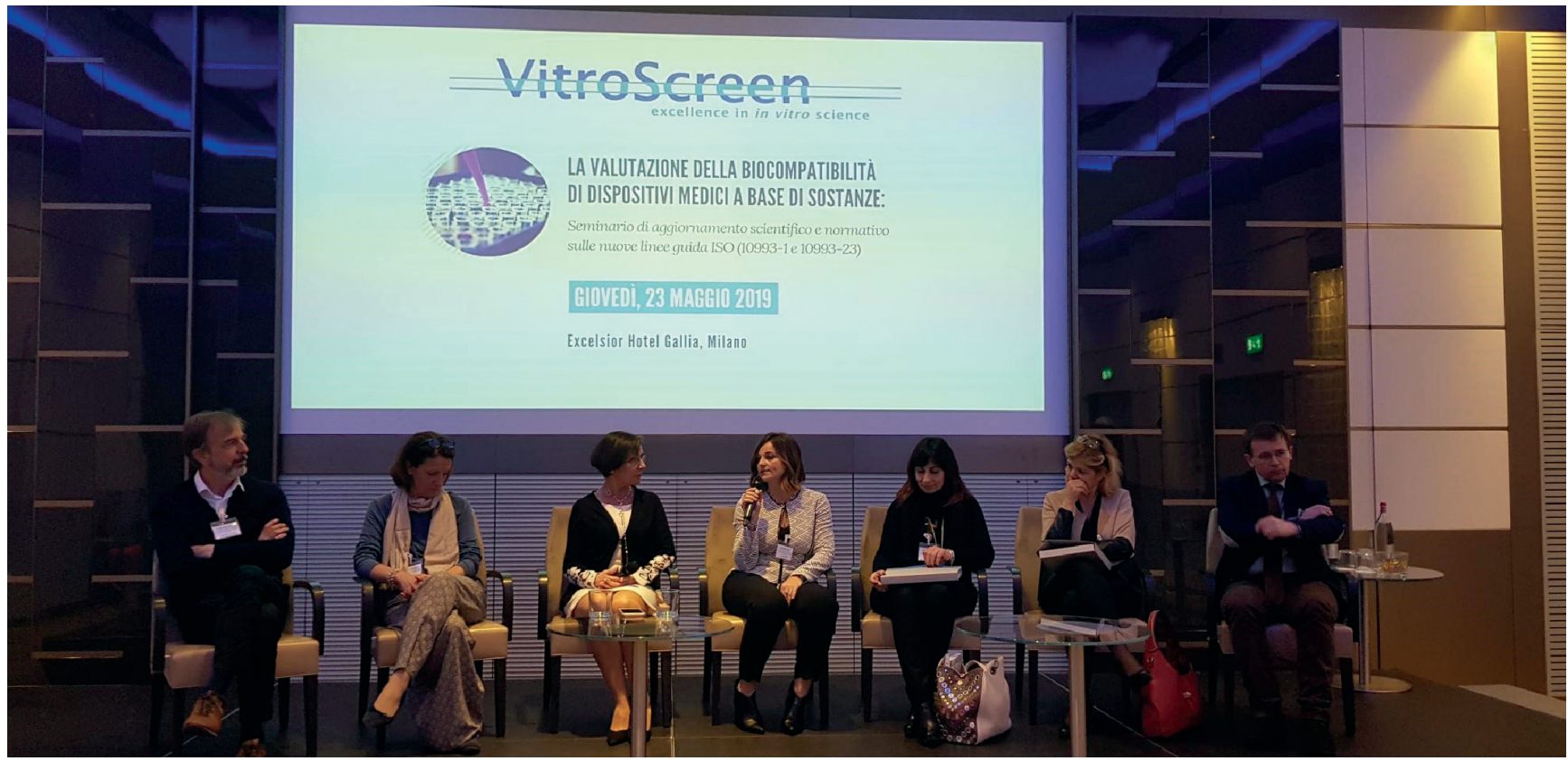

Round table discussion during the seminar on medical device biocompatibility evaluation

From left to right: Dr C. Pellevoisin (Episkin), Prof. E. Corsini (University of Milan), Dr M. Meloni (VitroScreen), Dr A. Frabetti (Kiwa Cermet), Dr R. Marcoaldi (ISS), Dr R. Feliciani (ISS), Dr M. Magni (ITALCERT).

extracts, while oil extracts will be challenging in the submerged cell cultures. For this case, 3D models based on reconstituted human epidermis should be fully explored. Accurate evaluation of relative skin sensitizing potency is still a challenge using in vitro methods, and these methods do not detect chemicals that act as adjuvants, enhancing an immune response to other chemicals to which a patient might be exposed, nor are they able to detect responses to antigens such as the plant proteins found in natural latex, which have been responsible for severe immune responses. Currently validated in vitro methods may be used to screen for dermal sensitization of medical devices, and thus reduce the need for animals, which still should be used to confirm negative results, saving time and money. However, their applicability to medical device extracts needs to be demonstrated before they can be used as routine tests.

More than 10 years ago, scientists called for a "paradigm shift in toxicology", away from animal testing toward human relevant, high-content, high-throughput integrated testing strategies to respond to the need of the chemical industry to test large numbers of chemicals and mixtures we are exposed to. VitroScreen's seminar drew the attention of the participants to how the transition to alternative toxicology has eventually reached the field of medical devices. The introduction of the new ISO 10993-23 standard is considered pivotal as a stand-alone, common and harmonized method to assess the irritation potential of medical device extracts without using animals. This milestone further opens the possibility to introduce new testing approaches based on human reconstructed tissues for which the presence of a 3D tissue structure allows the direct application, under realistic ex- posure conditions, of the product (e.g., gel, cream, etc.) onto the tissue where the device will exert its action. Different 3D human tissues are available (e.g., corneal, nasal, vaginal, etc.) and represent interesting models to assess the biocompatibility of devices acting on epithelia and mucosae other than epidermis.

A vibrant round table discussion at the end of the seminar confirmed the relevance of and the interest in the topic and allowed a stimulating interaction among the participants that can be summarized by the comment of Dr Amina Saaid, EU Technology and Innovation Manager at Sofibel Santé Beauté, Laboratoire Fumouze (FR): "We need more events like this: they should be organized in different places in Europe. After having listened to all the interventions, my feeling is that life science, and particularly in vitro science, is evolving at a faster speed than regulation, which is limiting us as industrials in innovating and marketing new products for the patients' wellbeing. It would be very helpful if industry experts, scientists and regulatory bodies worked together at the same speed and found the right way to mitigate these limitations."

\section{Marisa Meloni ${ }^{1}$, Laura Ceriotti ${ }^{1}$, Christian Pellevoisin ${ }^{2}$, Roberta Marcoaldi ${ }^{3}$, Roberta Feliciani ${ }^{3}$, Emanuela Corsini ${ }^{4}$} and Amina Saaid ${ }^{5}$

${ }^{1}$ VitroScreen, Milan, Italy; ${ }^{2}$ Episkin, Lyon, France; ${ }^{3}$ Istituto Superiore di Sanità, Rome, Italy; ${ }^{4}$ Laboratory of Toxicology, Department of

Environmental Science and Policy, Università degli Studi di Milano, Italy; ${ }^{5}$ Sofibel Laboratoire Fumouze, France

(laura.ceriotti@vitroscreen.com) 